\title{
Correlation Functions and the Uniqueness of the State in Classical Statistical Mechanics *
}

\author{
A. Lenard $\star \star$ \\ Institute for Advanced Study \\ Princeton, New Jersey, USA \\ Received October 31, 1972
}

\begin{abstract}
A general criterion is derived which assures the uniqueness of the state of a classical statistical mechanical system in terms of a given system of correlation functions. The criterion is $\sum_{k}\left(m_{k+j}^{A}\right)^{-1 / k}=\infty$ for all $j$ and all bounded sets $A$, where

$$
m_{k}^{A}=(k !)^{-1} \int_{A} \cdots \int_{A} \varrho_{k}\left(x_{1}, \ldots, x_{k}\right) d x_{1} \ldots d x_{k} .
$$
\end{abstract}

\section{Introduction}

In the older literature of classical statistical mechanics it was taken for granted, although not ex plicitly stated, that the sequence of correlation functions $\varrho_{n}, n=1,2,3, \ldots$, in their totality uniquely characterize the (statistical) state of the system to which they refer. That this is not the case in general was pointed out by Ruelle ${ }^{1}$. Thus, the problem arises of supplying criteria under which the uniqueness of the state is guaranteed. Such a criterion was already given in Ruelle's book, namely the existence of a positive constant $c$ such that $\left|\varrho_{n}\left(x_{1}, x_{2}, \ldots, x_{n}\right)\right| \leqq c^{n}$ for all $n$ and almost all values of the variables. Nevertheless, the question is interesting enough to merit more detailed investigation; the present paper is devoted to this task.

Before one can attack the problem it is necessary to specify precisely the mathematical set-up in whose context the question is posed. In a quite general manner, we do this as follows. We consider a space $X$ (the analogue of Gibbs's phase space) whose points $\xi$ are infinite "particle configurations" in a space $E$ (the "one particle space"). A natural measure theoretical structure is defined in $X$. A state of the system is taken to mean a probability measure $\mu$ over $X$. Since the correlation functions $\varrho_{n}$

\footnotetext{
* Research partially sponsored by the Office of Aerospace Research of the USAF under AFOSR Grant 70-1866C.

$\star \star$ Permanent address: Department of Mathematics, Indiana University, Bloomington, Indiana 47401, USA.

${ }^{1}$ Ref. [4], p. 103 and Exercise 4E.
} 
are densities with respect to the natural measure in $E$, a precise definition must be given for the quantities $\varrho(H)=\int \ldots_{H} \int \varrho_{n} d x_{1} \ldots d x_{n}$ where $H$ is some measurable set in $E^{n}$. We do this by showing that

$$
\varrho(H)=\int N_{H}(\xi) d \mu
$$

where, for each $H, N_{H}(\xi)$ is a measurable function over $X$ whose meaning can be specified by a certain combinational property of the configuration $\xi$ with respect to the set $H$. Our question is then: under what conditions on $\varrho$ is it guaranteed that (1) can have no more than one solution $\mu$ ?

The result of the investigation is a criterion stated in terms of quantities $m_{k}^{A}=\frac{1}{k !} \varrho\left(A^{k}\right)$ where $A$ is a bounded measurable set in $E$. We find that if the series

$$
\sum_{k=0}^{\infty}\left(m_{k+j}^{A}\right)^{-\frac{1}{k}}
$$

diverges for each $A$ and each integer $j$ then uniqueness for $\mu$ is assured. This criterion is much weaker than the one quoted above due to Ruelle; indeed, it is already satisfied when $\left|\varrho_{n}\left(x_{1}, \ldots, x_{n}\right)\right| \leqq c^{n} n^{2 n}$. The proof of our criterion depends on a remarkable theorem of Carleman [2] dealing with analytic functions.

\section{Mathematical Preliminaries}

To construct our mathematical set-up in the appropriate generality we start from a space $E$ which plays the role of the one-particle phase space (in practice $E=\mathbb{R}^{3}$ or $\mathbb{R}^{6}$, etc.). It is assumed to be a measurable space, and in addition the concept of boundedness is defined in it. The bounded sets in $E$ are a distinguished hereditary ${ }^{2}$ family of subsets closed under finite union formation. (If $E$ is a topological space, boundedness means having compact closure.) We assume that the class of measurable sets in $E$ is generated by the class of bounded measurable sets.

A configuration is defined to mean a finite or countably infinite system of points in $E$, considered without regard to order, and repetitions permitted. Thus a configuration is neither a set (whose elements are distinct, by definition), nor a sequence (where order matters). Configurations are generally denoted by the symbol $\xi$. The notation $\xi_{A}$ is used to denote the sub-configuration of $\xi$ consisting of the points of $\xi$ which are contained in the set $A \subseteq E$. We shall be concerned exclusively with locally finite configurations, i.e., with $\xi$ such that $\# \xi_{A}<\infty$ for every

\footnotetext{
${ }^{2}$ We use the measure theoretical terminology of Halmos [3].
} 
bounded $A$ (\# stands for "cardinal number of"). The set $X$ of all locally finite configurations plays the role of Gibbs's phase space. We now describe the construction which makes $X$ a measurable space: Let $A$ be any bounded measurable set in $E$ and $n$ any non-negative integer. Let $C_{n}^{A}=\left\{\xi \in X: \# \xi_{A}=n\right\}$. We define the class of measurable sets in $X$ as the smallest $\sigma$-algebra ${ }^{2}$ which contains all sets of the form $C_{n}^{A}$. A probability measure $\mu$ over $X$ is said to define a state of the system.

Among the measurable sets in $X$ there is a family of particular importance for us. Let $A_{1}, A_{2}, \ldots, A_{r}$ be disjoint bounded measurable sets in $E$, let $N$ be any set of $r$-tuples $\left(n_{1}, n_{2}, \ldots, n_{r}\right)$, and let

$$
C_{N}^{A_{1}, \ldots, A_{r}}=\left\{\xi \in X:\left(\# \xi_{A_{1}}, \ldots, \# \xi_{A_{r}}\right) \in N\right\} .
$$

The identity

$$
C_{N}^{A_{1}, \ldots, A_{r}}=\bigcup_{\left(n_{1}, \ldots, n_{r}\right) \in N} \bigcap_{j=1}^{r} C_{n_{j}}^{A_{j}}
$$

displays their measurability. It is easily verified that these sets ("cylinder sets") form an algebra, although not in general a $\sigma$-algebra. It is important that the restriction of a probability measure $\mu$ to these cylinder sets already guarantees the uniqueness of $\mu^{3}$. In other words, there can be at most one probability measure $\mu$ with a given system of numbers

$$
p_{n_{1}, \ldots, n_{r}}^{A_{1}, \ldots, A_{r}}=\mu\left\{\xi \in X: \# \xi_{A_{1}}=n_{1}, \ldots, \# \xi_{A_{r}}=n_{r}\right\},
$$

where the $A_{j}$ are arbitrary disjoint bounded measurable sets and the $n_{j}$ arbitrary non-negative integers.

Besides the space of configurations $X$, we also need the space $K$ of finite sequences of points in $E$. $K$ also contains the empty sequence. It is a disjoint union $K=\bigcup_{n=0}^{\infty} E^{n}$ (where $E^{0}$ is the set containing only the empty sequence, and $E^{n}=E \times E \times \cdots \times E$ with $n$ factors), and as such it is a measurable space in the natural manner. Any subset $H \cong K$ may be decomposed as a disjoint union $H=\bigcup_{n=0}^{\infty} H_{n}$ with $H_{n} \subseteq E^{n}$. If $H_{n}$ is bounded for all $n$ and $H_{n}=\emptyset$ for all sufficiently large $n$, we say that $H$ is bounded.

Let $\xi \in X$ and $H \cong K$. Suppose we enumerate the points of $\xi$ in some manner, say $\left(x_{1}, x_{2}, x_{3}, \ldots\right)$. For any finite sequence $\left(i_{1}, i_{2}, \ldots, i_{k}\right)$ of positive integers, the sequence $\left(x_{i_{1}}, x_{i_{2}}, \ldots, x_{i_{k}}\right)$ is a point of $K$. It may or may not belong to $H$. We denote by $N_{H}(\xi)$ the number of sequences $\left(i_{1}, i_{2}, \ldots, i_{k}\right)$, with $k$ arbitrary and the $i_{s}$ distinct, such that $\left(x_{i_{1}}, x_{i_{2}}, \ldots, x_{i_{k}}\right) \in H$. Note that the definition depends only on $\xi$ and not on the particular enumeration of its points, as is indicated by the notation. Note also that,

\footnotetext{
${ }^{3}$ Halmos [3], § 13, Theorem A.
} 
by definition, the empty sequence must also be considered in the counting the defines $N_{H}(\xi)$.

The number $N_{H}(\xi)$ may be viewed two ways. First, for fixed $\xi \in X$ it may be regarded as a set function $H \rightarrow N_{H}(\xi)$ defined for subsets $H \subseteq K$. Second, for fixed $H \subseteq K$ it may be regarded as a non-negative integer (including $+\infty$ ) valued function over the configuration space $X$.

From the first point of view the important fact is that $N_{H}(\xi)$ is completely additive, i.e., if $\left\{H_{j}\right\}$ is any family of disjoint subsets of $K$, then $\sum_{j} N_{H_{j}}(\xi)=N_{\cup_{j} H_{j}}(\xi)$. Thus, for every $\xi \in X, H \rightarrow H_{N}(\xi)$ is a measure. It follows ${ }^{4}$ that if $H_{1} \cong H_{2} \subseteq \ldots$, then $\operatorname{Sup}_{j} N_{H_{j}}(\xi)=N_{\cup} H_{j}(\xi)$, and if $H_{1} \supseteqq H_{2} \supseteqq \ldots$ with $N_{H_{1}}(\xi)<\infty$, then $\operatorname{Inf}_{j} N_{H_{j}}(\xi)=N_{\rho} H_{j}(\xi)$.

From the other point of view, the significant fact is that, roughly speaking, the nicer the set $H$ is, the nicer the function $\xi \rightarrow N_{H}(\xi)$. For instance, if $H=A_{1}^{k_{1}} A_{2}^{k_{2}} \ldots A_{r}^{k_{r}}$ with disjoint $A_{j}$, then we have the explicit formula.

$$
N_{H}(\xi)=\frac{\# \xi_{A_{1}} !}{\left(\# \xi_{A_{1}}-k_{1}\right) !} \cdots \frac{\# \xi_{A_{r}} !}{\left(\# \xi_{A_{r}}-k_{r}\right) !},
$$

as one verifies directly from the definition. The general regularity property of $\xi \rightarrow N_{H}(\xi)$ is stated in the following proposition.

Theorem 1. If $H \subseteq K$ is a bounded measurable set, then the function $\xi \rightarrow N_{H}(\xi)$ is finite valued and measurable.

Proof. Since $H$ is bounded, we have $H=\bigcup_{n} H_{n}$ where the union is finite and $H_{n} \subseteq A^{n}$ for some bounded $A \subseteq E$. We have then

$$
N_{H}(\xi)=\sum_{n} N_{H_{n}}(\xi) \leqq \sum_{n} N_{A^{n}}(\xi)=\sum_{n} \frac{\# \xi_{A} !}{\left(\# \xi_{A}-n\right) !}<\infty .
$$

This shows the finite valuedness. To prove that $\xi \rightarrow N_{H}(\xi)$ is measurable we proceed in steps. This is obvious in case $H$ is of special form $H=A_{1}^{k_{1}} A_{2}^{k_{2}} \ldots A_{r}^{k_{r}}$ as displayed by (2.3), and of course also when $H$ is a finite union of such "rectangles". These finite unions form a ring of sets $^{5}$, say $\mathscr{R}$. Now let $\mathscr{F}$ be the class of those subsets $H \subseteq K$ for which $\xi \rightarrow N_{H}(\xi)$ is measurable, thus $\mathscr{R} \cong \mathscr{F}$. We now show that $\mathscr{F}$ is a monotone class, i.e., that if $H_{1} \subseteq H_{2} \cong \ldots$ with $H_{j} \in \mathscr{F}$ then $\bigcup_{j} H_{j} \in \mathscr{F}$, and also if $H_{1} \supseteqq H_{2} \supseteqq \ldots$ with $H_{j} \in \mathscr{F}$ then $\bigcap_{j} H_{j} \in \mathscr{F}$. Take the first case. We have seen that $N_{\cup_{j}}(\xi)=\operatorname{Sup}_{j} N_{H_{j}}(\xi)$. But a pointwise increasing limit of

\footnotetext{
${ }^{4}$ Halmos [3], §9, Theorems D and E.

${ }^{5}$ Halmos [3], $\$ 4$.
} 
measurable functions is measurable; thus $\bigcup_{j} H_{j} \in \mathscr{F}$. The case of a decreasing sequence follows by the analogous reasoning. Now, it is a fact of general measure theory that if a monotone class $\mathscr{F}$ contains a ring $\mathscr{R}$ then it also contains the $\sigma$-ring generated by the ring ${ }^{6}$. But the $\sigma$-ring generated by $\mathscr{R}$ in our case is, by definition, the class of all measurable subsets of $K$.

Q.E.D.

\section{Correlation Functions and the Measure $\varrho$}

Let $\mu$ be the probability measure over $X$ defining a state of the system. For every bounded measurable $H \subseteq K$ we write

$$
\varrho(H)=\int_{X} N_{H}(\xi) d \mu .
$$

According to Theorem 1 this definition makes sense, and $\varrho(H) \geqq 0$. It can, of course, happen that $\varrho(H)=\infty$. If $\varrho(H)<\infty$ for all bounded measurable $H$, we say that $\mu$ possesses finite correlations. In the following this condition will always be assumed, even if not mentioned explicitly. The set function $H \rightarrow \varrho(H)$ is a measure, for it is clearly finitely additive with respect to $H$, and $\sigma$-additivity follows easily from the bounded convergence theorem ${ }^{7}$. We call it the correlation measure associated with the given state $\mu$.

This terminology is justified by the relation $\varrho$ bears to the socalled correlation functions in classical statistical mechanics. Recall that in the conventional situation (where $E$ is ordinary Euclidean space $\mathbb{R}^{v}$ ) the correlation function $\varrho_{n}$ is defined by the statement that $\varrho_{n}\left(x_{1}, x_{2}, \ldots, x_{n}\right)$ $d x_{1} d x_{2} \ldots d x_{n}$ is the probability for finding particles in each of the $n$ volume elements $d x_{j}$ (simultaneously) ${ }^{8}$. From the strict mathematical point of view, $\varrho_{n}$ is therefore not a function but must be regarded as a measure, and instead of the above "definition" one should rather specify the meaning of the set function obtained by integrating the correlation "functions" over arbitrary measurable sets. We claim that the proper definition is as follows: For every bounded measurable $H=\bigcup_{n} H_{n}, H_{n} \subseteq E^{n}$,

$$
\varrho(H)=\sum_{n} \int \underset{H_{n}}{\ldots} \varrho_{n} d x_{1} \ldots d x_{n} .
$$

To justify this statement let us proceed formally and assume that for every bounded measurable set $A \subseteq E$ the probability that there are precisely $n$ particles (i.e., points of the configuration $\xi$ ) in $A$ and they

6 Halmos [3], §6, Theorem B.

7 Halmos [3], § 26, Theorem D.

8 Ruelle [4], § 4.1.1. 
are in the volume elements $d x_{1}, \ldots, d x_{n}$ is $\pi_{n}^{A}\left(x_{1}, \ldots, x_{n}\right) d x_{1} \ldots d x_{n}$. The correlation functions are then given, for arguments in $A$, by the formula

$$
\varrho_{k}\left(x_{1}, \ldots, x_{k}\right)=\sum_{s=0}^{\infty} \frac{1}{s !} \int_{A} \ldots \int_{A} \pi_{k+s}^{A}\left(x_{1}, \ldots, x_{k}, y_{1}, \ldots, y_{s}\right) d y_{1} \ldots d y_{s}
$$

the factor $(s !)^{-1}$ accounting for the fact that the points of a configuration are unlabelled. Noting that the probability for finding $m$ particles in $A$ is

$$
\mu\left(C_{m}^{A}\right)=\frac{1}{m !} \int_{A} \cdots \int_{A} \pi_{m}^{A}\left(x_{1}, \ldots, x_{m}\right) d x_{1} \ldots d x_{m},
$$

we obtain from (3.3)

$$
\begin{aligned}
\int_{A} \cdots \int_{A} \varrho_{k}\left(x_{1}, \ldots, x_{k}\right) d x_{1} \ldots d x_{k} & =\sum_{m=k}^{\infty} \frac{m !}{(m-k) !} \mu\left(C_{m}^{A}\right) \\
& =\int_{X} \frac{\# \xi_{A} !}{\left(\# \xi_{A}-k\right) !} d \mu=\int_{X} N_{A^{k}}(\xi) d \mu
\end{aligned}
$$

This verifies the equality (3.2) for the special case $H=A^{k}$. It is just as easy to verify if for $H=A_{1}^{k_{1}} A_{2}^{k_{2}} \ldots A_{r}^{k_{r}}$ where the $A_{j}$ are disjoint bounded measurable subsets of $E$. By additivity (3.2) is thus seen to hold for the class of subsets $H \leqq K$ which are finite unions of such rectangles (i.e., the ring $\mathscr{R}$, cf. above, end of Section 2). But this establishes (3.2) generally because of the uniqueness ${ }^{3}$ of the extension of a measure from $\mathscr{R}$ to the $\sigma$-ring of all measurable sets in $K$.

In the following we make no use of (3.2), nor even assume the existence of the densities $\varrho_{n}$, but regard it only as a heuristic justification for dealing with the measure $\varrho$ which is defined by (3.1).

But (3.1) may also be viewed from another point of view, namely, as an equation for the unknown $\mu, \varrho$ being given. If so viewed, it gives rise to two fundamental problems. First, under what conditions on $\varrho$ does there exist any solution? Second, under what conditions on $\varrho$ can there be no more than one solution? The two problems of existence and uniqueness have very different mathematical character. Here we shall be concerned only with the second of these problems which is the exact formulation of the question raised in the Introduction. The author hopes to come back to the existence problem in a later publication.

Our Eq. (3.1) somewhat resembles a classical problem in Analysis, namely the Stieltjes Moment Problem. There a measure $\sigma$ is sought, say on the positive real line, such that

$$
c_{k}=\int_{0}^{\infty} t^{k} d \sigma(t)
$$


where the $c_{k}(k=0,1,2, \ldots)$ are given numbers ${ }^{9}$. The resemblance between our problem and the Moment Problem is shown more explicitly if we change the latter slightly. First, instead of the functions $t^{k}$ we consider the functions $\left(\begin{array}{l}t \\ k\end{array}\right)=\frac{t(t-1) \ldots(t-k+1)}{k !}$, second, we restrict attention to measures $\sigma$ which are concentrated on the integers. Thus, instead of (3.6), we consider the similar problem

$$
m_{k}=\sum_{n=0}^{\infty}\left(\begin{array}{l}
n \\
k
\end{array}\right) p_{n},
$$

where the $m_{k}(k=0,1,2, \ldots)$ are given numbers, and $p_{n}=\sigma(\{n\})$ form components of the unknown probability vector. The analogy with (3.1) is brought out if we consider special sets $H$, namely rectangles $H=A_{1}^{k_{1}} A_{2}^{k_{2}} \ldots A_{r}^{k_{r}}$, where the $A_{j}$ are disjoint bounded measurable sets in $E$. Using (2.3) and the notation (2.2) we have

$$
\begin{aligned}
m_{k_{1}, \ldots, k_{r}}^{A_{1}, \ldots, A_{r}} & =\frac{1}{k_{1} ! k_{2} ! \ldots k_{r} !} \varrho\left(A_{1}^{k_{1}} A_{2}^{k_{2}} \ldots A_{r}^{k_{r}}\right) \\
& =\sum_{n_{1}, \ldots, n_{r}}\left(\begin{array}{l}
n_{1} \\
k_{1}
\end{array}\right)\left(\begin{array}{l}
n_{2} \\
k_{2}
\end{array}\right) \ldots\left(\begin{array}{l}
n_{r} \\
k_{r}
\end{array}\right) p_{n_{1}, n_{2}, \ldots, n_{r}}^{A_{1}, A_{2}, \ldots, A_{r}}
\end{aligned}
$$

which is quite analogous to (3.7). Because we are interested in the question of uniqueness for $\mu$ we emphasize that, as has already been remarked in Section 2, a unique determination of the family of numbers $p_{n_{1}, \ldots, A_{r}}^{A_{1}, \ldots, n_{r}}$ implies a unique $\mu$. Thus we are concerned with a uniqueness criterion for the system of equations (3.8).

Our principal result is the following

Theorem 2. If for all bounded measurable $A \cong E$, and all nonnegative integers $j, \sum_{k=0}^{\infty}\left(m_{k+j}^{A}\right)^{-\frac{1}{k}}$ diverges, then $\mu$ is uniquely determined by $\varrho$.

Before proving the theorem, let us make a few remarks.

From the formula $m_{k}^{A}=\int\left(\begin{array}{c}\# \xi_{A} \\ k\end{array}\right) d \mu$ it is evident that $m_{k}^{A}$ is a nondecreasing function of the set $A$. Therefore, the series occurring in our theorem certainly diverges for some $A$ if it diverges for some $A^{\prime} \supseteqq A$. Thus the theorem deals in effect only with the asymptotic behavior of $m_{k}^{A}$ for large $A$. Of course, it also deals only with the behavior of $m_{k}^{A}$ as $k \rightarrow \infty$.

\footnotetext{
${ }^{9}$ See for instance Akhiezer [1].
} 
Here are a few simple corollaries of the theorem. If

$$
\operatorname{Sup}_{0 \leqq k<\infty} \frac{\left(m_{k}^{A}\right)^{\frac{1}{k}}}{k}<\infty
$$

for every bounded $A$, then the condition of the theorem is satisfied. This is easily checked: If $m_{k}^{A} \leqq\left(c_{A} k\right)^{k}$, then $\left(m_{k+j}^{A}\right)^{-\frac{1}{k}} \geqq\left[c_{A}^{(k+j)}\right]^{-1-\frac{j}{k}}$, and for sufficiently large $k$, say $(k+j)^{-\frac{j}{k}} \geqq 1 / 2,\left(m_{k+j}^{A}\right)^{-\frac{1}{k}} \geqq \frac{\text { constant }}{k+j}$, showing the divergence of the series.

If $\varrho$ is representable in terms of correlation functions $\varrho_{n}$ by the formula (3.2), we may convert our criterion into a simple condition on the $\varrho_{n}$. Writing $\left\|\varrho_{n}\right\|$ for the essential supremum of $\left|\varrho_{n}\left(x_{1}, x_{2}, \ldots, x_{n}\right)\right|$ for $x_{1}, x_{2}, \ldots, x_{n} \in E$, the condition reads

$$
\left\|\varrho_{n}\right\| \leqq\left(c n^{2}\right)^{n},
$$

where $c$ is a constant. For this implies $m_{k}^{A} \leqq \frac{\left(v_{A}\right)^{k}}{k !}\left\|\varrho_{k}\right\| \leqq \frac{\left(c v_{A}\right)^{k}}{k !} k^{2 k}$, where $v_{A}=\int_{A} d x$, and so Sup $\frac{1}{k}\left(m_{k}^{A}\right)^{-\frac{1}{k}} \leqq e c v_{A}$.

It may be worth pointing out that there is a much stronger condition than the one given in the theorem, a condition that is, however, particularly easy to apply in concrete instances. This condition is that the power series $\sum_{k=0}^{\infty} m_{k}^{A} z^{k}$ have a positive radius of convergence for every bounded $A$. Indeed, this is equivalent to $\operatorname{Lim} \operatorname{Inf}\left(m_{k}^{A}\right)^{-\frac{1}{k}}=\operatorname{Lim} \operatorname{Inf}\left(m_{k+j}^{A}\right)^{-\frac{1}{k}}>0$; thus, the series occurring in the theorem has terms positively bounded away from zero in this case.

\section{Proof of Theorem 2}

Omitting the indices, we denote by $\bar{p}$ and $p$ two solutions of the system of Eqs. (3.8). Assuming that they are not identical, we have bounded measurable sets $A_{1}, \ldots, A_{r} \subseteq E$ such that $\bar{p}_{n_{1}, \ldots, n_{r}}^{A_{1}, \ldots, A_{r}}$ and $\underline{p}_{n_{1}, \ldots, n_{r}}^{A_{1}, \ldots, A_{r}}$ are not equal as functions of the integers $n_{1}, \ldots, n_{r}$. Let us introduce generating functions

$$
\phi_{s}=\sum_{n_{1}, \ldots, n_{r}}\left(\begin{array}{l}
n_{1} \\
k_{1}
\end{array}\right) \ldots\left(\begin{array}{l}
n_{s} \\
k_{s}
\end{array}\right) z_{s+1}^{n_{s}+1} \ldots z_{r}^{n_{r}} p_{n_{1}, \ldots, n_{r}}^{A_{1}, \ldots, A_{r}}
$$

associated with solutions $p$ of (3.8). These are analytic for $\left|z_{s+1}\right|, \ldots,\left|z_{r}\right|<1$. We have then for $s=0,1,2, \ldots, r$ two such functions $\bar{\phi}_{s}$ and $\underline{\phi}_{s}$ associated 
with the two solutions $\bar{p}$ and $\underline{p}$. We note that, by hypothesis, $\bar{\phi}_{0}=\phi_{0}$; on the other hand, $\bar{\phi}_{r} \equiv \phi_{r}$ since for $s=r$ the right hand side of (4.1) is just the given quantity in (3.8). We now fix $s$ as the smallest number so that $\bar{\phi}_{s} \equiv \phi_{s}$ but $\bar{\phi}_{s-1}=\phi_{s-1}$. Let $\psi=\bar{\phi}_{s-1}-\phi_{s-1}$; we view it as a function of the complex variable $z=z_{s}$, with the other variables so fixed that $\psi \neq 0$.

We need an elementary estimate that follows from the identity

$$
(1+z)^{n}=\sum_{j=0}^{k-1}\left(\begin{array}{l}
n \\
j
\end{array}\right) z^{j}+\left(\begin{array}{l}
n \\
k
\end{array}\right) z^{k} k \int_{0}^{1}(1-t)^{k-1}(1+t z)^{n-k} d t .
$$

Denote the last term by $R_{n, k}(z)$. If $|1+z|<1$ and $0 \leqq t \leqq 1$, then also $|1+t z| \leqq 1$. Therefore $\left|k \int_{0}^{1}(1-t)^{k-1}(1+t z)^{n-k} d t\right| \leqq k \int_{0}^{1}(1-t)^{k-1} d t=1$, and so

$$
\left|R_{n, k}(z)\right| \leqq\left(\begin{array}{l}
n \\
k
\end{array}\right)|z|^{k}
$$

whenever $|1+z|<1$. In the identity (4.2) we replace $1+z$ by $z$, and substitute it into the power series for $\psi(z)$, choosing $k=k_{s}$ an arbitrary integer. One now observes that the first $k$ terms of (4.2) yield zero, since

$$
\sum \bar{p}_{n_{1}, \ldots \ldots, A_{r}}^{A_{1} \ldots}\left(\begin{array}{l}
n_{1} \\
k_{1}
\end{array}\right) \ldots\left(\begin{array}{l}
n_{s-1} \\
k_{s-1}
\end{array}\right)\left(\begin{array}{l}
n_{s} \\
k_{s}
\end{array}\right) z_{s+1}^{n_{s+1}} \ldots z_{r}^{n_{r}}
$$

is identical to the corresponding sum with $\bar{p}$ replaced by $\underline{p}$, by our choice of $s$. From the estimate (4.3) one obtains then

$$
\begin{aligned}
|\psi(z)| & \leqq|z-1|^{k_{s}} \sum\left(\bar{p}_{n_{1}, \ldots, n_{r}}^{A_{1}, \ldots, A_{r}}+\underline{p}_{n_{1}, \ldots, n_{r}}^{A_{1}, \ldots, A_{r}}\right)\left(\begin{array}{l}
n_{1} \\
k_{1}
\end{array}\right) \ldots\left(\begin{array}{l}
n_{s-1} \\
k_{s-1}
\end{array}\right)\left(\begin{array}{l}
n_{s} \\
k_{s}
\end{array}\right) \\
& =2 m_{k_{1}, \ldots, k_{s}}^{A_{1}, \ldots, A_{s}}|z-1|^{k_{s}}
\end{aligned}
$$

(Here we used the identity

$$
\sum_{n_{s}+1, \ldots, n_{r}} p_{n_{1}, \ldots, n_{r}}^{A_{1}, \ldots, A_{r}}=p_{n_{1}, \ldots, n_{s}}^{A_{1}, \ldots, A_{s}}
$$

which follows from the definition (2.2).) Let $k_{1}+\cdots+k_{s-1}=j$. Use the obvious inequality

$$
\left(\begin{array}{l}
n_{1} \\
k_{1}
\end{array}\right) \cdots\left(\begin{array}{l}
n_{s-1} \\
k_{s-1}
\end{array}\right)\left(\begin{array}{l}
n_{s} \\
k_{s}
\end{array}\right) \leqq\left(\begin{array}{c}
n_{1}+\cdots+n_{s} \\
j+k_{s}
\end{array}\right),
$$

as well as the identity

$$
\sum_{n_{1}+\cdots+n_{s}=n} p_{n_{1}, \ldots, n_{s}}^{A_{1}, \ldots, A_{s}}=p_{n}^{A},
$$


where $A=A_{1} \cup \cdots \cup A_{s}$. From (4.4) it follows then that

$$
|\psi(z)| \leqq 2 m_{j+k}^{A}|z-1|^{k} .
$$

Here $z$ is subject only to $|z|<1$, and $k$ is any non-negative integer. Our hypothesis about the two not identical solutions $\bar{p}$ and $p$ has resulted in an analytic function $\psi$, not identically zero, and satisfying the infinite system of inequalities (4.5).

The following beautiful proposition becomes now relevant.

Theorem (Carleman [2]). Let $f(z)$ be analytic for $|z|<1$, and let it satisfy inequalities of the form $|f(z)| \leqq\left(\beta_{k}|z-1|\right)^{\lambda_{k}}, k=1,2, \ldots$, where $0<\lambda_{1}<\lambda_{2}<\cdots \rightarrow \infty, 0<\beta_{1}, \beta_{2}, \ldots$, and $\sum_{k=1}^{\infty} \frac{\lambda_{k}-\lambda_{k-1}}{\beta_{k}}$ diverges. Then $f$ is identically zero ${ }^{10}$.

We let $f=\psi, \lambda_{k}=k$ and $\beta_{k}=\left(2 m_{j+k}^{A}\right)^{1 / k}$. The inequalities in the statement of the theorem are just (4.5). Thus $\psi$ vanishes identically. Therefore $\bar{p}=\underline{p}$, the solution of (3.8) is unique, and so is the state $\mu$.

Q.E.D.

It may be of methodological interest that the above proof is patterned closely after the uniqueness proof of Carleman ${ }^{11}$ concerning the Stieltjes Moment Problem which yields a criterion very similar to the one in our Theorem 2.

\section{References}

1. Akhiezer, N.I.: The classical moment problem and some related questions in analysis. Edinburgh: Oliver and Boyd 1965.

2. Carleman,T.: Les fonctions quasi analytiques, Chapter III. Paris: Gauthier-Villars 1926.

3. Halmos, P. R.: Measure theory. New York: Van Nostrand 1950.

4. Ruelle, D.: Statistical mechanics, rigorous results. New York: Benjamin 1969.
A. Lenard
Department of Mathematics
Indiana University
Bloomington 47401, USA

${ }^{10}$ Carleman proves a slightly different version of the theorem, namely one dealing with analytic functions in a half-plane. Our statement follows from his by a suitable fractional linear transformation.

11 Carleman [2], Chapter VIII. 